\title{
Radial velocities with CRIRES ${ }^{\star} \star \star$ Pushing precision down to $5-10 \mathrm{~m} / \mathrm{s}$
}

\author{
P. Figueira ${ }^{1}$, F. Pepe ${ }^{1}$, C. H. F. Melo ${ }^{2}$, N. C. Santos ${ }^{3}$, C. Lovis ${ }^{1}$, M. Mayor ${ }^{1}$, D. Queloz ${ }^{1}$, A. Smette ${ }^{4}$, and S. Udry ${ }^{1}$ \\ 1 Geneva Observatory, University of Geneva, 51 Ch. des Maillettes, 1290-Sauverny, Switzerland \\ e-mail: pedro.figueira@unige.ch \\ 2 European Southern Observatory, Karl-Schwarzschild-Strasse 2, 85748 Garching bei Muenchen, Germany \\ 3 Centro de Astrofísica, Universidade do Porto, Rua das Estrelas, 4150-762 Porto, Portugal \\ 4 European Southern Observatory, Alonso de Córdova 3107, Vitacura, Casilla 19001, Santiago 19, Chile
}

Received 12 June 2009 / Accepted 10 December 2009

\begin{abstract}
With the advent of high-resolution infrared spectrographs, radial relocity (RV) searches enter into a new domain. As of today, the most important technical question to address is which wavelength reference is the most suitable for high-precision RV measurements. In this work we use atmospheric absorption features as wavelength reference on CRIRES data obtained on two programs and three different targets. We analyzed the data from the TW Hya campaign again, reaching a dispersion of about $6 \mathrm{~m} / \mathrm{s}$ on the RV standard on a time scale of roughly 1 week. We confirm that there is a low-amplitude RV signal on TW Hya itself, with an amplitude roughly 3 times smaller than the one reported at visible wavelengths. We present RV measurements of Gl 86 as well, showing that our approach is capable of detecting the signal induced by a planet and correctly quantifying it. Our data show that CRIRES is capable of reaching an RV precision of less than $10 \mathrm{~m} / \mathrm{s}$ on a time scale of one week. The limitations of this particular approach are discussed, along with the limiting factors on RV precision in the IR in a general way. The implications of this work on the design of future dedicated IR spectrographs are addressed as well.
\end{abstract}

Key words. stars: planetary systems - infrared: stars - instrumentation: spectrographs - methods: observational techniques: radial velocities

\section{Introduction}

The discovery of a hot Jupiter orbiting 51 Peg by Mayor \& Queloz (1995) triggered the quest for extrasolar planets. This campaign made use of the radial velocity technique (henceforth $\mathrm{RV})$, by which the presence of a planetary companion is inferred by the wobble it induces on the parent star. Several dedicated surveys have employed this method, concentrating in the beginning on solar-type stars, the most favorable target for high-precision measurements. However, it soon became clear that extrasolar planets were ubiquitous around these hosts. Longing for a better understanding of formation processes, planetary searches started to diversify, targeting young (Sozzetti et al. 2006; Setiawan et al. 2007) and evolved (Lovis \& Mayor 2007; Sato et al. 2008), lowmass (Bonfils et al. 2004; Endl et al. 2008) and massive stars (Hatzes et al. 2005; Galland et al. 2005).

Recent technological developments allowed more precise spectrographs to be built, such as HARPS (Mayor et al. 2003), and reduction and analysis methods have been perfected through the years (Lovis \& Pepe 2007). As of today, HARPS yields the most accurate RV measurements, with sub-m/s precision,

* Based on observations taken at the VLT (Paranal), under programs 280.C-5064(A) and 60.A-9051(A), and with the CORALIE spectrograph at the Euler Swiss telescope (La Silla).

$\star \star$ Tables 2 and 3 are only available in electronic form at the CDS via anonymous ftp to cdsarc.u-strasbg.fr $(130.79 .128 .5)$ or via http://cdsweb.u-strasbg.fr/cgi-bin/qcat?]/A+A/511/A55 allowing for a succession of ground-breaking detections of the lightest planets known (Lovis et al. 2006; Mayor et al. 2009).

Both the search for more exquisite stellar hosts and the race for the lightest planets point towards $M$ dwarfs. These stars, which are the most abundant in the Universe, are also the lightest ones. Since the RV variation induced by a planet on a star scales with $M^{-2 / 3}$, the amplitude of the effect induced on an M star is significantly larger. As an example, a planet of an identical mass at the same distance from the stellar host produces an RV variation with an amplitude $\sim 3$ times larger on an M5 star than on a G2 star. The drawback is that since they are much colder, M dwarfs are much fainter in optical wavelengths. The RV survey of light-mass stars points then towards the exploration of a new wavelength domain, the IR, where the luminosity of these objects peaks.

As RV searches started to target young and active stars, another advantage of the IR became apparent. RV signals can be created by surface inhomogeneities, such as stellar spots (Queloz et al. 2001). Since the nature of these phenomena can bypass current diagnosis methods like the bisector analysis (see, for instance, Desort et al. 2007), a planetary origin can be assigned to a periodic variation that is, in fact, of stellar origin. By observing in the IR, as opposed to the visible wavelengths, one observes in a domain where the contrast between stellar spots and the stellar disk is reduced. Since the spot/star contrast ratio is at the root of the RV signal detected, the amplitude of the latter is reduced as well (Martín et al. 2006; Huélamo et al. 2008; Prato et al. 2008). By comparing RV drawn from optical and IR spectrographs, one 
can assign the planetary or stellar nature to an RV signal in an unambiguous way.

However, the exploration of the RV in the IR is in its infancy, and many problems still need to be tackled. In this work we concentrate on describing a precise wavelength calibration technique for the IR domain. In Sect. 2 we discuss the difficulties of wavelength calibration in the IR and present the principles of our technique. In Sect. 3 we describe CRIRES and the observations, and how we applied our approach. Section 4 presents an outline of the data reduction and analysis. Section 5 presents our results. Section 6 discusses these results and their implications for the design of spectrographs dedicated to RV measurements in the IR. We finish by stating our conclusions in Sect. 7.

\section{Wavelength references in the IR}

To detect small-amplitude RV signals (i.e., of $\mathrm{m} / \mathrm{s}$ ) a simultaneous wavelength reference must be employed. In the visible wavelengths, the Th-Ar emission lamps and the $I_{2}$ absorption cell have proved to yield a precision of better than $1 \mathrm{~m} / \mathrm{s}$ (Mayor et al. 2009) and $3 \mathrm{~m} / \mathrm{s}$ (Howard et al. 2009), respectively. As impressive as they are, the transfer of this knowledge to the IR domain is not straightforward. The density of Th-Ar lines is much lower in the IR than in the visible, making it difficult to use. On top of that a stable spectrograph is required, since the lamp lines do not follow the same instrumental profile (IP) variations as the science targets (they pass through different optical paths). The more frequent gas-cell approach does not provide a better solution, because no known gas creates a dense absorption forest in a wide wavelength range in the IR. Several hybrid alternatives were put forward to attempt to solve this problem, such as gas cell plus emission lamp or coupling of different gas cells. For a detailed description of the problem and proposed solutions the reader is referred to the work of Mahadevan \& Ge (2009).

A different approach can be taken by using one of the peculiarities of the IR: the absorption of light by the Earth's atmosphere. Our atmosphere is mainly composed of molecular species, whose rotational and vibrational features correspond to near-IR wavelengths. Usually seen as a nuisance, they can be used to our advantage. In some narrow wavelength domains, the presence of well-defined, sharp, and deep lines can provide for a good local wavelength solution. While this is only possible locally, it is well suited for the current high-resolution IR spectrographs, characterized by a small simultaneous wavelength coverage, typically of $\lambda / 50-70$. As a free and always present gas cell, using atmospheric features as a wavelength reference has to be assessed before venturing into other alternatives.

However, our "gas-cell" suffers from a particular problem: the lines it imprints on the spectra depend on the atmospheric conditions. While some of lines' properties are expected to remain constant, others, such as depth, vary at a level of several \%. It is then very hard to apply the typical deconvolution technique (Butler et al. 1996), which assumes a detailed knowledge of the absorbing spectra to calculate the IP and correct for it. While several dedicated models have been developed (Bailey et al. 2007) to address this problem, there is no evaluation of the impact of the remaining residuals on RV, and it is arguably bigger than the $\mathrm{m} / \mathrm{s}$ level. As a matter of fact, the only campaign that employed the deconvolution approach in the IR in the more standard way, Blake et al. (2007), reported a precision of $300 \mathrm{~m} / \mathrm{s}$ and stated that a precision down to $100 \mathrm{~m} / \mathrm{s}$ was within reach. However, this result should be taken with caution, as it was derived for $\mathrm{M}$ dwarfs, a demanding stellar host.
We decided to depart from this approach by avoiding the overlapping between target and reference features. While the spectra of the star and of the atmosphere are simultaneous in the sense that they are obtained at the same moment, the lines are separated spectroscopically and can be fitted independently. The center of each line is thus determined in a straightforward way (for instance, the atmospheric lines' depth variation has no impact on the measured RVs). The IP variations that affect stellar lines affect reference lines as well. If the same fitting procedures are employed on both line sets, the effect cancels out.

Several notable experiments on the use of atmospheric absorption features were made in the eighties, of which we highlight the results of Balthasar et al. (1982), Smith (1982), and Caccin et al. (1985). Working with $\mathrm{O}_{2}$ lines, these authors could reach a precision of $5 \mathrm{~m} / \mathrm{s}$. The value is made even more relevant by their using different RV determination methods (different instrumentation, different line fitting approaches, etc.), now believed to be obsolete when compared with the cross correlation function (Baranne et al. 1996) or the deconvolution method. Snellen (2004) used the same principle on $\mathrm{H}_{2} \mathrm{O}$ lines and reached a precision of $5-10 \mathrm{~m} / \mathrm{s}$ on UVES data. This shows that the accuracy of our wavelength reference is at least of $5 \mathrm{~m} / \mathrm{s}$, making it a valuable option for us.

\section{Observations}

\subsection{CRIRES}

CRIRES (CRyogenic InfraRed Echelle Spectrograph) is the new ESO IR spectrograph mounted on the Unit Telescope 1 (UT1, Antu) of the European Southern Observatory Very Large Telescope (Kaeufl et al. 2004). The main optical elements consist of a prism acting as a pre-disperser and a 31.6 lines $/ \mathrm{mm}$ echelle grating. The instrument provides for a resolution of up to 100000 when used with a $0.2^{\prime \prime}$ slit. It operates in the range 960-5200 $\mathrm{nm}$ with an instantaneous wavelength coverage of $\sim \lambda / 50$. The spectra is imaged on a detector mosaic, composed of four Aladdin III detectors $(4096 \times 512$ pixel $)$ with a gap of $\sim 250$ pix between the chips. As a consequence, the instantaneous wavelength coverage is much narrower than for optical cross-dispersed spectrographs, such as HARPS, HIRES or UCLES. Adaptive Optics (MACAO - Multi-Applications Curvature Adaptive optics) can be used to optimize the signalto-noise ratio and the spatial resolution.

\subsection{The observing set-up}

At the time of observations, CRIRES had 200 possible observing set-ups, most of which overlapped ${ }^{1}$. To select the most suitable set-up for precise RV, we need to decide which one carries the higher content of useful spectral information. We stress here that by "useful" we mean domains where both reference - i.e. atmospheric - and target - i.e. stellar - spectral features are not superposed.

We used solar NSO/Kitt Peak FTS data (Livingston \& Wallace 1991; Wallace et al. 1993) to simulate CRIRES spectra. To do so, we convolved the spectra with an IP that we assumed as being Gaussian and defined as a function of resolution (fixed at $R=100000$ ), and re-sampled it on the detector. This

\footnotetext{
1 Since May 2009, there are 282 standard wavelength setups available. The standard wavelength setups are designed to provide a complete coverage with no contamination by adjacent orders. In addition, users can select a free wavelength setting.
} 
procedure was applied for the Sun spectra, atmospheric spectra, and the product of the two. To access the RV uncertainty measured on a sun-like spectrum using the atmosphere as reference, we calculated the final RV uncertainty as the squared sum of the estimated error on the atmospheric lines' RV and on the stellar lines' RV. It is important to stress that the objective was not to obtain an estimation of the value of attainable precision in absolute terms but in a relative way between the different settings.

To estimate the precision on each of the components, two different methods were employed: 1) a simple rule of thumb that states that the inverse of RV accuracy $\epsilon \propto S / N \cdot \bar{I} \cdot \sqrt{n_{1}}$, where $S / N$ is the signal-to-noise ratio of the spectra, $\bar{I}$ the average depth of absorption lines, and $n_{1}$ the number of lines in the spectra; 2) the quality factor described in Bouchy et al. (2001), which quantifies the spectral information present on each spectra.

It is clear that the obtained precision depends heavily on the useful spectral domain. In our particular case, the superposition of telluric and stellar spectra can deem several zones as useless for RV calculation. To take this effect into account, we proceeded as follows. We used an automated algorithm to identify stellar lines in the simulated spectra (Sousa et al. 2007). From these we selected only those stellar lines that were not superimposed on atmospheric lines bigger than a fixed threshold, and vice-versa. We added a Doppler shift in the interval $[-30,+30] \mathrm{km} \mathrm{s}^{-1}$ in order to reproduce the line shift created by Earth's movement around the Sun (for the case of a star that has an average RV of 0 relative to us). Lines that were found to be superposed after this step were discarded as well. We varied the detection and superposition thresholds at the level of $1-5 \%$, a reasonable value for high $S / N$ spectra. As the superposition of a relatively small line on the measured one can already introduce an RV shift of several hundreds of meters per second, it was mandatory to keep the superposition threshold low.

At the end of this procedure two zones were identified as the most promising:

- the $H$ band, at $1.6 \mu \mathrm{m}$ - the telluric absorption is dominated by $\mathrm{CO}_{2}$ lines, while other absorbers have negligible absorption, smaller than $1 \%$. The $\mathrm{CO}_{2}$ spectrum is easily identified and provides for very regular wavelength anchors, with around 30 deep lines - from 10 to $60 \%$ - on several detectors. The stellar spectra provides deep lines, which, while not being very numerous, allow for an average contrast of around $30 \%$;

- the $K$ band, at $2.32 \mu \mathrm{m}$ - the atmospheric absorption shows features of several molecules, with $\mathrm{CH}_{4}$ being the most prominent. The stellar lines depth is below $10 \%$, but the high number (around 50) compensates for it. This was the setting chosen by Blake et al. (2007).

We found out that the detection and superposition thresholds were the parameters that led to the predominance of one out of the two domains. For values higher than $5 \%$, the $H$ band zone dominates. Figure 1 depicts the output of this simulation, with the spectral content increasing along the y axis. Over-plotted is the squared root of the average stellar flux, in arbitrary units as well. The final precision depends on the product of these two factors and the objective is to maximize it. The two bumps represent the wavelength domains where atmospheric transmission becomes so low that the the precision estimators fall along with stellar flux.

The main qualitative difference between the two domains in what concerns spectral information can be summarized as follows: while in the $K$ band the spectral information is extracted

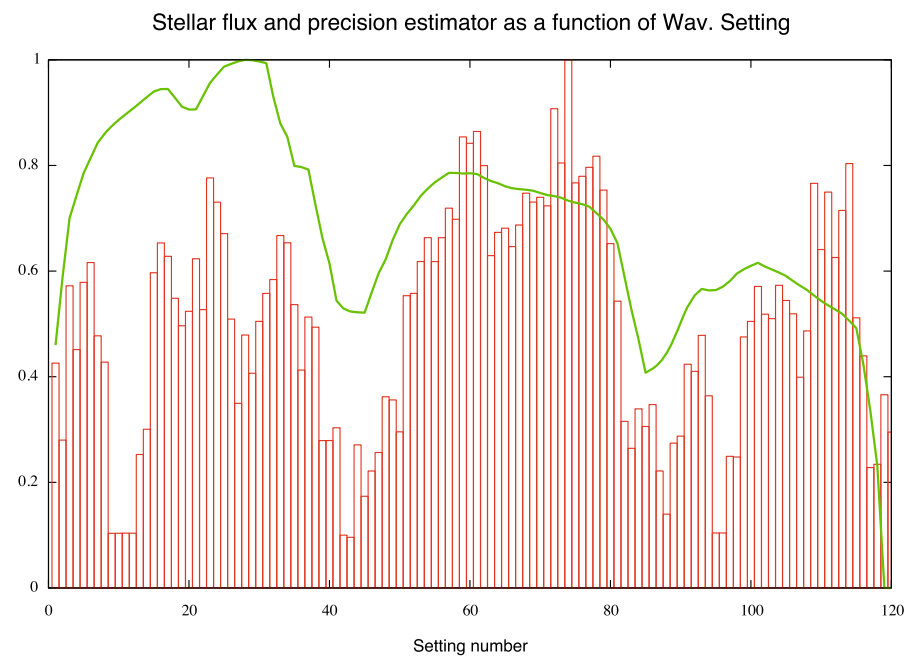

Fig. 1. Precision as a function of wavelength setting, in arbitrary units, as described in Sect. 3.2 (histogram, red in the electronic version). Over-plotted is the squared root of the average solar flux, as seen by CRIRES in a given wavelength setting, in arbitrary units (thick line, green in the electronic version). All setting short-wards of $2.4 \mu \mathrm{m}$ are depicted. $H$ band favorable zone is located between settings 60-75 and $K$ band's around 110-115. Note that settings where numbered as a function of increasing wavelength reference, following the order presented in CRIRES manual.

from more numerous and shallower atmospheric lines, in the $H$ band it is extracted from less numerous, deeper lines. The crowding of lines in the $K$ band increases the risk of mutual pollution between the two sets of lines (telluric and stellar), which makes precise measurements more difficult in the $K$ band than in $H$ band.

The final choice was the atmospheric transmission window in the $H$ band, in particular the setting (36/1/i). This setting provides for deep, sharp atmospheric lines of $\mathrm{CO}_{2}$ in detectors 1,2 and 4. Special attention was given to image the highest number of deep stellar lines in detectors 1 and 2, as the blaze function provides for a much higher transmission in these two. The detector 1 is completely covered by a comb of 22 lines, detector 2 has 14 lines on it, and detector 4 images 20 lines. Deep lines, with relative depths of $10-50 \%$, provide for a homogeneous complete coverage of the first detector; in the second one the depths range from 40 to $10 \%$ for the first two thirds of the detector and are smaller than $10 \%$ for the last third; finally, lines with a relative depth of $10-55 \%$ cover $90 \%$ of the fourth detector. It is very important to note that there are no other absorbers than $\mathrm{CO}_{2}$, providing for both an unambiguous identification of spectral features and a well-defined continuum around the sharp reference lines. For illustration purposes, in Fig. 2 we plot the atmospheric transmission measured by observing the telluric standard HD 96146.

The simplified study presented here does not intend to be the last word on the choice setting for CRIRES for RV studies. The optimal setting depends not only on the aforementioned superposition parameters chosen but also on the stellar spectra, the mean RV of the target and even the line identification algorithms chosen to pinpoint the lines present in each spectrum. More generally than that, it depends on the RV determination method. Our objective here is to provide for spectrally separated stellar and atmospheric lines, so that the spectral information carried by these well-defined lines can be fully exploited.

To conclude we note that the choice of employing the atmosphere as a wavelength reference was prompted by very practical 

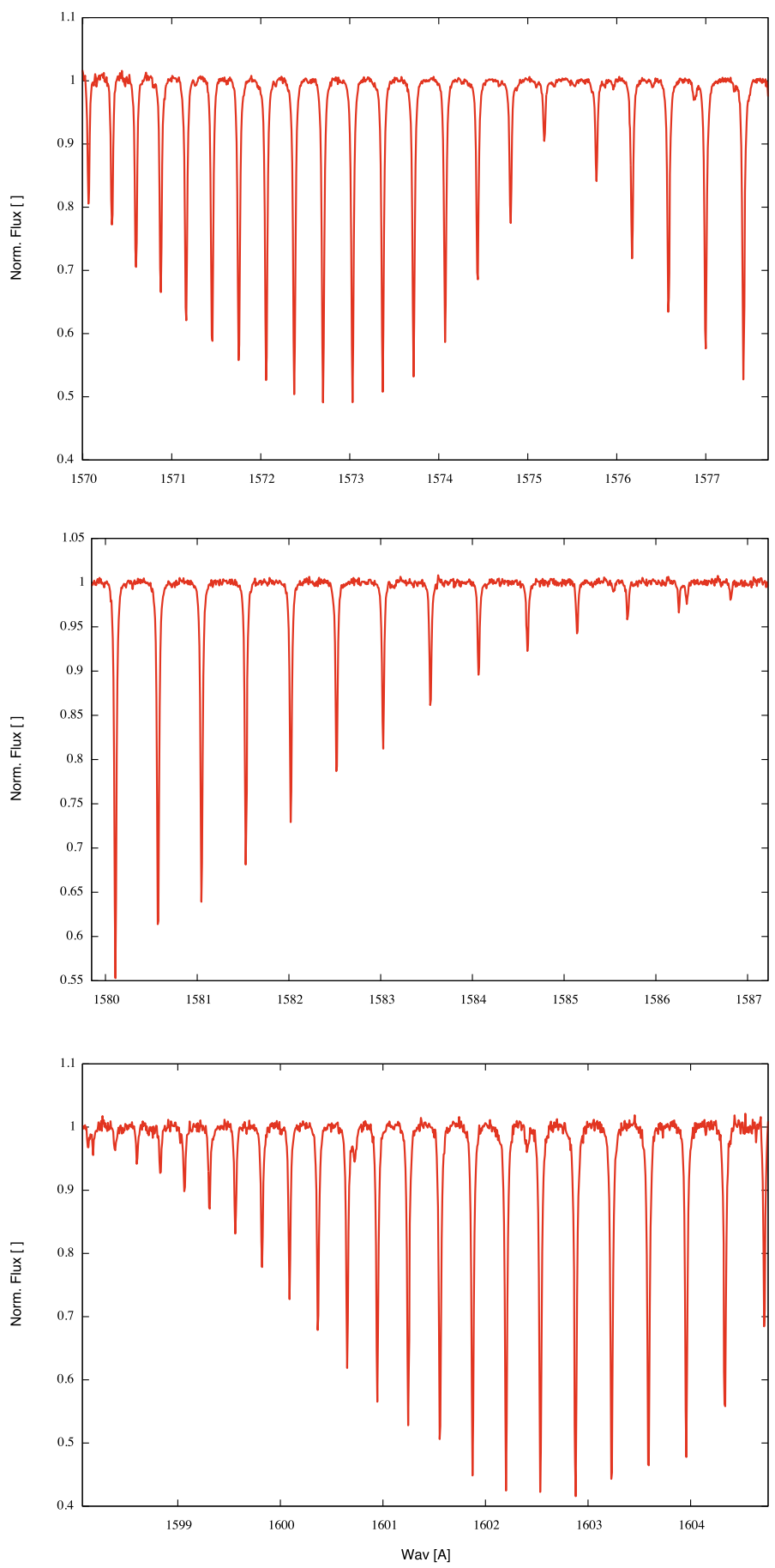

Fig. 2. Atmospheric transmission at the chosen observing setting, for the detectors 1 (top), 2 (middle), and 4 (bottom). These spectra were obtained by co-adding and then normalizing the telluric standard exposures taken during the first night.

issues. At the time of our observations, there was no CRIRES gas cell available capable of providing deep absorption lines shortwards of $2.5 \mu \mathrm{m}$.

An entrance slit of $0.2^{\prime \prime}$ was chosen, allowing for a resolution of $\sim 100000$. To reduce guiding errors (expected to have $1-1$ translation on the lines profile and thus on the measured RV) two cautionary measures were taken. First of all, no AO was used, to make sure that the star PSF was spread and larger than the slit width. On top of that, observations during bad seeing periods were requested. The objective was to provide enough photons to the slit viewer/guiding camera to ensure a good centering of the star on the slit and to reduce photo-center effects on RV. While these effects affect both star and reference in the same way, minimizing their cause is the best way of ensuring a precise correspondence between the two.

\subsection{Observations}

The TW Hya campaign was performed under Director Discretionary Time (DDT), with program number 280.C5064(A). The extensively studied TW Hya star was observed five times, from the 22 to 28 February 2008, promptly followed by an RV velocity standard, HD 108309. This star has been observed by HARPS high-precision programs and is known to be stable down to $1.1 \mathrm{~m} / \mathrm{s}$. Between the science target and the RV standard the instrumental set-up remained fixed, and no instrumental changes were allowed for. The objective was to track possible zero-point drifts of our wavelength calibration, which could have either an instrumental or atmospheric origin. The observations were made using the wav. setting $(36 / 1 / i)$, characterized by the reference wavelength $1592.7 \mathrm{~nm}$ (defined as the wavelength at the center of detector 3 ).

Originally, a set of $10 \mathrm{RV}$ measurements of TW Hya on preferably consecutive nights was requested. However, due to scheduling difficulties aggravated by multiple instrument interventions, the period ended with only 5 out of the 10 measurements being taken.

The CRIRES calibration plan comprises an RV monitoring of radial velocity standard stars with the objective of studying CRIRES' stability. From this data we recovered the observations on G1 86. The observations were made on the 5, 9, 17 and 18 January 2008 with a slit of $0.3^{\prime \prime}$ in the setting $(36 / 1 / n)$, and no AO was used.

Table 1 summarizes the properties of the observations.

\section{Data reduction and analysis}

Data were reduced using a custom pipeline written in IRAF's $\mathrm{CL}^{2}$ (Tody 1993). The procedures were tailored to CRIRES spectra using first the science verification data and later the data sets analyzed in this paper. It provides for automated dark and nonlinearity corrections (using the nonlinearity coefficients provided by ESO), as well as flagging and replacement of bad pixels. The images were corrected from sensitivity variations by dividing by a flat-fielding corrected from the blaze function effect. The nodding pairs were mutually subtracted and the orders' tracing was fitted using cubic splines. An optimal extraction algorithm was employed for extracting the spectra (Horne 1986). The objective of creating a dedicated pipeline is not only to optimize IRAF tunable knobs to provide for an optimized reduction but also to ensure a consistent and homogeneous reduction for all spectra.

The computation of RV from extracted spectra was performed using a Python and FORTRAN pipeline heavily based on HARPS' DRS (Data Reduction Software). The $\mathrm{CO}_{2}$ lines' central wavelength was provided by HITRAN database (Rothman et al. 2005), with an accuracy of 5-50 m/s. The high $S / N$ of the spectra and the wide separation between spectral lines allowed us to fit telluric lines easily and determine their centers.

\footnotetext{
2 IRAF is distributed by the National Optical Astronomy Observatories, which are operated by the Association of Universities for Research in Astronomy, Inc., under cooperative agreement with the National Science Foundation.
} 
Table 1. The summary of the observation details on the RV std, TW Hya, and Gl 86, with all NDIT set to 1.

\begin{tabular}{lccccc}
\hline \hline Object & DIT [s] & \# of Nodding pairs & Reference Wavelength [nm] & Slit width ["] & Set-up comments \\
\hline RV std & 45 & 2 & 1592.7 & 0.2 & No AO \& No Gas-Cell \\
TW Hya & 180 & 2 & 1592.7 & 0.2 & No AO \& No Gas-Cell \\
Gl 86 & 30 & 3 & 1588.4 & 0.3 & No AO \& No Gas-Cell \\
\hline
\end{tabular}

For each frame, the wavelength solution was obtained by fitting a third degree polynomial that matches the fitted centers with the theoretical wavelengths. Earth's RV in its orbit around the Sun was calculated using the ephemerides of Bretagnon \& Francou (1988), which also allowed the computation of barycentric julian dates. By using the Earth's RV one can transpose the wavelength solution to the Solar System barycenter. RVs are then calculated by cross-correlating the spectra against a template mask as described in Baranne et al. (1996). In our case we used weighted masks (Pepe et al. 2002) to take the impact of photon noise on lines of different depth into account. We created two sets of masks: one from PHOENIX stellar atmosphere models (Barman et al. 2005) and the other by measuring the position and depth of each stellar line directly on the co-added reduced spectra. The center of the cross-correlation function was determined by fitting a Gaussian function. We note that we used the same line fit for both our reference and our science target lines. IP variations, which affect the two line sets in the same way, were fitted by the same procedure, and its impact on RVs cancels out.

As described in Sect. 2, extreme caution is needed to reduce mutual pollution between stellar and atmospheric spectra to a minimum. By knowing Earth's RV and the approximate RV of the target ${ }^{3}$, one can calculate the approximate position of each telluric and stellar line and discard those that blend. While this might lead to a significant loss of lines, it greatly reduces the systematic error introduced by blended lines. As a result the photon noise calculations on the remaining lines provide for a realistic assessment of the spectral information carried and achievable precision.

Because the RV is computed for each nodding position spectrum and detector, the RV determination is independent for each frame, as the omnipresent reference lines are fitted independently, and their centers are used to construct a dedicated wavelength solution. The dispersion of the RV within the nodding cycle then allows for the direct calculation of the uncertainty on the averaged value for the nodding cycle. The error bar is simply the r.m.s. of the independent nodding measurements divided by the square root of the number of measurements.

Along with each RV calculation, the bisector inverse slope was computed on the stellar cross-correlation function following the procedure described in Queloz et al. (2001).

\section{Results}

Owing to the very steep blaze function, the low $S / N$ of TW Hya spectra collected in detector 4 made atmospheric line fitting impossible. On the other hand, the low number of unblended stellar lines in detector 2 combined with the lower count number led to a very high photon noise on the CCF function. For the sake of consistency and to allow a simple and robust comparison, the standard RV presented were calculated only using the first detector as well. In Fig. 3 we plot the spectra of HD 108309 ,

\footnotetext{
3 Note that the RV variations eventually induced by planets are about $100 \mathrm{~m} / \mathrm{s}$ and thus much smaller than the line's FWHM. As a consequence, these small amplitude fluctuations can be ignored at this stage.
}

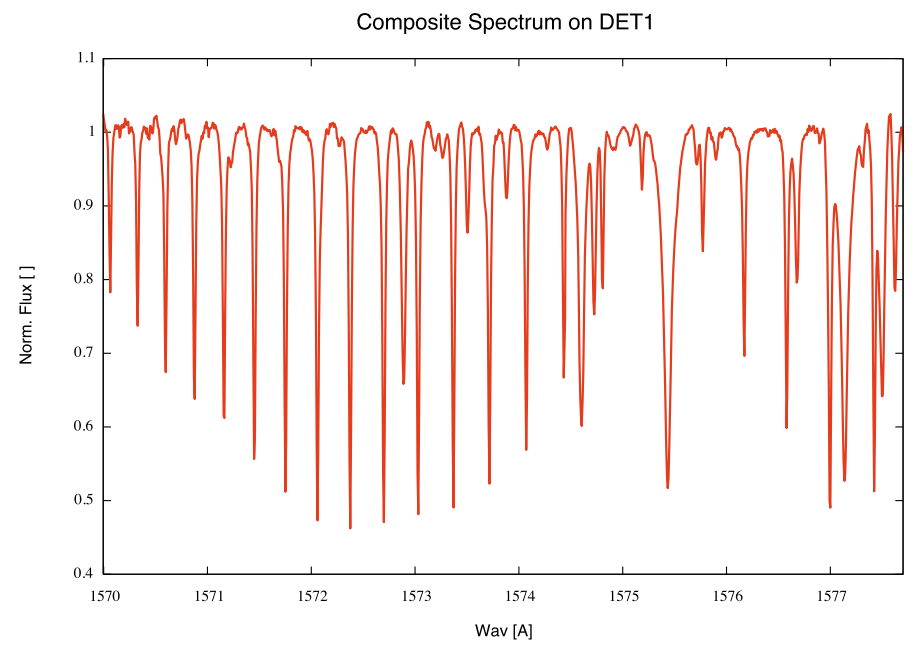

Fig. 3. Spectrum of RV standard, HD 108309, as seen on detector 1 . These spectra are the result of the stacking and normalization of the 4 nodding spectra obtained during the first night.

on detector 1, for illustration purposes. Out of the 22 lines, 14 unblended lines were used to calculate the wavelength solution; these lines have an average depth of $35.1 \pm 12.8 \%$ and $F W H M=3.11 \pm 0.31$ pxl.

All RV values presented here were obtained with a template mask built from the reduced CRIRES spectra. Results derived from masks built from PHOENIX models yielded a lower precision. The dispersion of the RV standard measurements, the most reliable precision indicator among our measurements, is of $\sim 25 \mathrm{~m} / \mathrm{s}$ for PHOENIX masks. The reason for this difference is not known.

The RV measurements of TW Hya and the associated standard star are presented in Table 2 and plotted in Fig. 4. The dispersion of the standards' RV measurements is compatible with the error bars, while that is not the case for TW Hya. Simultaneous optical RV determinations were performed by CORALIE to back up CRIRES observations, already described in detail in Huélamo et al. (2008). TW Hya IR RVs are not compatible with the orbit drawn from optical RV measurements, with an amplitude of roughly $200 \mathrm{~m} / \mathrm{s}$, defined using CORALIE (Baranne et al. 1996) data and reproducing Setiawan et al. (2008) data perfectly. To test the hypothesis of a smaller RV semiamplitude in the IR, we left this quantity and the systemic velocity as free parameters, fixing all others. The fit delivers a semiamplitude of $80.50 \pm 6.83 \mathrm{~m} / \mathrm{s}$ with an $\mathrm{rms}(\mathrm{O}-\mathrm{C})$ around the fit of $7.93 \mathrm{~m} / \mathrm{s}$. The fit is overplotted on the data.

The RV measurements of G1 86 are presented in Table 3 and Fig. 5. The orbit presented in Queloz et al. (2000) is superposed on the data. At first, only the systemic velocity and the $T_{0}$ were allowed to vary. The reason for leaving $T_{0}$ as a free parameters comes from the error propagation from the 2000 orbit to 2008 leading to a very large error on this parameter. The uncertainty adds up to almost 8 days, which is roughly half of the orbital period. The agreement between the published curve and 
A\&A 511, A55 (2010)

Table 2. The RV calculated per nodding position for the RV standard, HD 108309, and TW Hya, and estimated photon noise of each exposure.

\begin{tabular}{|c|c|c|c|c|c|c|c|}
\hline RVstd & MJD [d] & $\mathrm{RV}\left[\mathrm{km} \mathrm{s}^{-1}\right]$ & $\mathrm{Ph}$. Noise $[\mathrm{m} / \mathrm{s}]$ & TW Hya & MJD [d] & $\mathrm{RV}\left[\mathrm{km} \mathrm{s}^{-1}\right]$ & $\mathrm{Ph}$. Noise $[\mathrm{m} / \mathrm{s}]$ \\
\hline & 2454519.805826 & 30.3699 & 10.5 & & 2454519.795056 & 12.1094 & 20.6 \\
\hline & 2454519.806647 & 30.3600 & 10.1 & & 2454519.797602 & 12.0837 & 19.1 \\
\hline & 2454519.807307 & 30.3452 & 10.1 & & 2454519.799998 & 12.1662 & 19.4 \\
\hline & 2454519.808129 & 30.2390 & 10.7 & & 2454519.802579 & 12.0965 & 18.9 \\
\hline & 2454522.666534 & 30.3146 & 16.8 & & 2454522.655881 & 12.2158 & 32.9 \\
\hline & 2454522.667368 & 30.3778 & 16.4 & & 2454522.658416 & 12.1857 & 26.0 \\
\hline & 2454522.668039 & 30.3330 & 16.2 & & 2454522.660823 & 12.2122 & 29.3 \\
\hline & 2454522.668861 & 30.3402 & 16.7 & & 2454522.663370 & 12.2435 & 29.9 \\
\hline & 2454523.728265 & 30.2983 & 14.4 & & 2454523.716034 & 12.0599 & 26.1 \\
\hline & 2454523.729098 & 30.3300 & 14.7 & & 2454523.718569 & 12.0379 & 23.8 \\
\hline & 2454523.729770 & 30.3419 & 16.4 & & 2454523.720965 & 12.1981 & 25.9 \\
\hline & 2454523.730592 & 30.3281 & 15.7 & & 2454523.723534 & 12.0555 & 25.8 \\
\hline & 2454524.749738 & 30.3288 & 13.4 & & 2454524.734402 & 12.0436 & 24.6 \\
\hline & 2454524.750571 & 30.3301 & 12.3 & & 2454524.736948 & 12.1082 & 22.8 \\
\hline & 2454524.751242 & 30.3526 & 11.6 & & 2454524.739344 & 12.0788 & 34.0 \\
\hline & 2454524.752064 & 30.3389 & 12.5 & & 2454524.742249 & 12.0935 & 26.1 \\
\hline & 2454525.733788 & 30.3323 & 11.5 & & 2454525.713207 & 12.2017 & 22.2 \\
\hline & 2454525.734610 & 30.3690 & 10.0 & & 2454525.715765 & 12.1959 & 18.0 \\
\hline & 2454525.735270 & 30.3403 & 9.6 & & 2454525.718161 & 12.1969 & 19.3 \\
\hline & 2454525.736092 & 30.3208 & 9.8 & & 2454525.720730 & 12.1501 & 19.7 \\
\hline
\end{tabular}
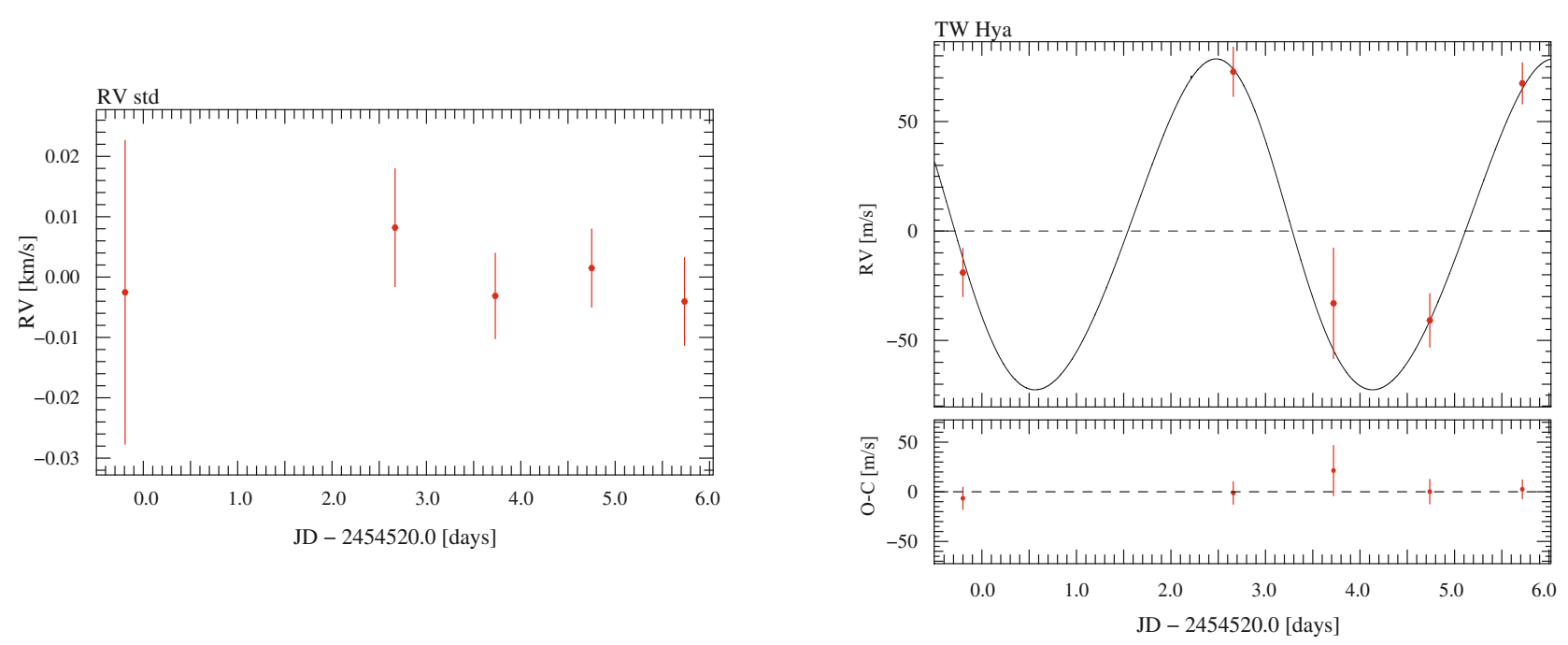

Fig. 4. RV variation of the standard star (left) and TW Hya (right) as a function of time. The error bars are drawn from the dispersion of the $N=4$ nodding positions, divided by $2(\sqrt{N})$, as described in Sect. 4. The optical orbit, with a different $K$ (as described in Sect. 5) is overplotted on the points.

CRIRES measurements is shown by the small dispersion around it, only $5.4 \mathrm{~m} / \mathrm{s}$. Alternatively, one can fix $T_{0}$ and let $P$ vary: the $P$ remains unchanged within error bars, and the dispersion of the residuals remains roughly the same. We recently obtained several CORALIE measurements on this star that, together with the 2000 data, allowed us to independently determine the $T_{0}$. This procedure delivered a value compatible, within error bars, with the one provided by CRIRES points alone. There is a measurable drift caused by the presence of an outer companion (Eggenberger et al. 2003) and the determination of the $T_{0}$ at the moment of CRIRES data (when there are no CORALIE points) is affected by the shape of the unknown orbit. In either case, this $T_{0}$ value can be used to fit an orbit in the CRIRES data, leaving only the systemic velocity as the free parameter. This delivers a dispersion of $16 \mathrm{~m} / \mathrm{s}$, which, while bigger, shows the agreement between the orbit and CRIRES data.

We can estimate the precision expected on computing the cross-correlation function on each spectrum, drawn from the

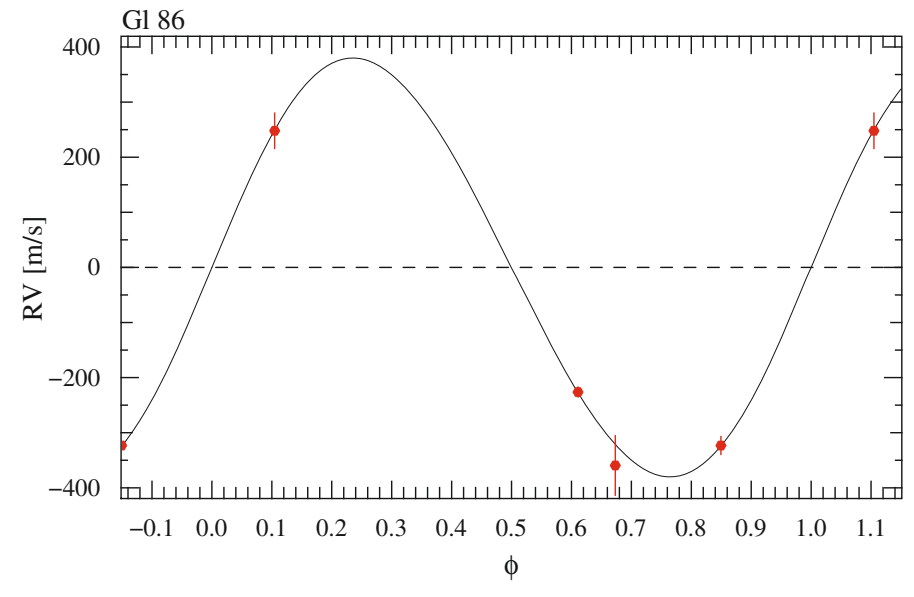

Fig. 5. Phase-folded plot of Gl 86 Rv measurements. The error bars presented are drawn from the dispersion of the six nodding positions, divided by $\sqrt{6}$, as discussed in Sect. 4 . 
Table 3. The RV per nodding position for Gl 86, and associated photon noise.

\begin{tabular}{cccc}
\hline \hline Gl 86 & MJD [d] & RV $\left[\mathrm{km} \mathrm{s}^{-1}\right]$ & Ph. Noise $[\mathrm{m} / \mathrm{s}]$ \\
\hline 2454470.519076 & 55.6681 & 18.1 \\
2454470.519713 & 55.5957 & 16.7 \\
2454470.520199 & 55.6705 & 18.3 \\
2454470.520847 & 55.6617 & 20.4 \\
2454470.521345 & 55.6703 & 17.6 \\
2454470.521969 & 55.7322 & 17.8 \\
2454474.547247 & 56.3486 & 8.7 \\
2454474.547930 & 56.2396 & 8.4 \\
2454474.548508 & 56.2105 & 8.7 \\
2454474.549145 & 56.1806 & 8.5 \\
2454474.551020 & 56.1226 & 8.5 \\
2454474.551656 & 56.3237 & 8.1 \\
2454482.535667 & 55.7555 & 12.9 \\
2454482.536315 & 55.7780 & 11.6 \\
2454482.536813 & 55.7990 & 11.9 \\
2454482.537461 & 55.7627 & 14.3 \\
2454482.537970 & 55.7263 & 13.6 \\
2454482.538618 & 55.7589 & 12.0 \\
2454483.517437 & 55.8188 & 11.9 \\
2454483.518085 & 55.5305 & 11.8 \\
2454483.518571 & 55.6780 & 12.2 \\
2454483.519207 & 55.7346 & 12.2 \\
2454483.519694 & 55.4173 & 13.3 \\
2454483.520342 & 55.6021 & 11.5 \\
\hline
\end{tabular}

photon noise alone. To do so we use the method presented in Bouchy et al. (2001). We consider the particular case of a Gaussian absorption line and apply it to the stellar lines present in the correlation mask (the unblended ones actually used in the correlation, so of interest to us). This yields the precision on each individual spectrum, i.e., on each nodding position. To calculate the error on the averaged night value we need to divide it by the square root of the number of independent nodding positions.

We can then compare three different estimators of the reached precision:

- the weighted dispersion of the night-averaged RV points external dispersion;

- the error on the averaged night value, given by dispersion on the nodding cycle RV values divided by $\sqrt{N}$ - intra-night dispersion;

- the average photon error estimator value (as described above) divided by $\sqrt{N}$ - photon noise.

Table 4 presents all these results. These errors were estimated in such a way that they can be easily compared, as they provide for alternative ways of looking at the same quantity. In the cases of TW Hya and Gl 86, these can be compared with the dispersion around the fit.

\section{Discussion}

In our previous work (Huélamo et al. 2008), we reached a precision of $35 \mathrm{~m} / \mathrm{s}$ over a time span of roughly 1 week. This value was obtained in relative RV: science target - RV standard. This procedure allowed for a correction of the systematics still present in the data after our first reduction. Seifahrt \& Käufl (2008), a contemporary study, reached a precision of $20 \mathrm{~m} / \mathrm{s}$ on one night. A dedicated effort was made to track systematics and understand their possible causes. As a result we could now improve the analysis procedure to extract the RV in an optimized way, leading to a significant increase in precision. We no longer need to calculate relative RV to correct for systematics. The RV standard dispersion is now close to photon noise, showing that there are no systematics present down to $5-10 \mathrm{~m} / \mathrm{s}$. The same is valid for TW Hya and Gl $86(\mathrm{O}-\mathrm{C})$ residuals, which show a similar dispersion.

The RV standard displays a variation of less than $6 \mathrm{~m} / \mathrm{s}$ (rms) over 1 week. The probability that a dispersion of $5.77 \mathrm{~m} / \mathrm{s}$ or lower is obtained on 5 points belonging to a distribution with $\sigma=6.48 \mathrm{~m} / \mathrm{s}$ (the estimated photon noise) is $58.2 \%$. If we consider 5 points drawn from a distribution characterized by $\sigma=7.66 \mathrm{~m} / \mathrm{s}$ (the measured internal dispersion), the probability then drops to $40.8 \%$, but it is still high. We calculated the probability of drawing a dispersion of $5.77 \mathrm{~m} / \mathrm{s}$ from distributions with $\sigma$ from 5 to $10 \mathrm{~m} / \mathrm{s}$. The results, presented in Table 5, show that we cannot exclude that the intrinsic dispersion of our distribution is slightly larger than the measured one. As a result we consider the attained precision, in a conservative way, as being $6-8 \mathrm{~m} / \mathrm{s}$.

It is interesting to compare the values of intranight dispersion with photon-noise estimations, for the three targets. When measuring the intranight dispersion, one considers not only the photon noise but also the effect of systematic errors on RV. A straightforward example of such are the errors induced by nodding and locating the target at different positions in the slit, known to have two slit jaws that are not perfectly parallel. While the values are very similar for the RV standard and TW Hya, that is not the case for Gl 86. A possible cause is the use of a larger entrance slit, and the consequent introduction of additional sources of error, such as stellar photocenter variations.

The RV values for the standard and TW Hya are compatible, within error bars, with the previously published values. This is mostly caused by the comparatively large dispersion announced previously $(35 \mathrm{~m} / \mathrm{s})$ and error bars with even larger amplitude $(30-60 \mathrm{~m} / \mathrm{s})$. However, given the higher precision of the measurements, a low-amplitude signal now emerges now from the noise. As previously discussed in (Huélamo et al. 2008), a lower amplitude signal in the IR than in the optical is expected if a spot is responsible for the RV variations instead of a planet. The previously presented tests stand as well. One can calculate the orbit parameters from optical observations. The probability that the IR RV points are drawn from this orbit by adding an error comparable to the instrumental precision is below $1 \times 10^{-3} \%$, showing that the spot theory prevails.

The RV determinations for Gl 86 match the orbit as established by the announcement paper. It is important to stress that for this fit the semi-amplitude, $P, e$, and $\omega$ were fixed on the published values in Queloz et al. (2000). This shows that our method yields the correct value for RV signals, reproducing the bona-fide planet detected at optical wavelengths.

Given the very few points for both TW Hya and Gl 86, the dispersion around the respective orbits should be taken with caution. At this level the number of free parameters approaches the number of points and the noise contribution is fitted as well, leading to a $\chi^{2}<1$. As a representation of this, one can use the TW Hya data in a slightly different way. One may use the RV values obtained for each nodding position, with error bars given by the photon-noise estimation. The fit delivers, within error bars, the same orbit parameters ( $K$ and $\gamma$ ), a dispersion of the residuals of $35 \mathrm{~m} / \mathrm{s}$ and a $\chi^{2}=1.63$, showing that the error bars are underestimated by photon noise alone. At this point our objective is merely to show that the RV points are drawn from the orbits. They do not define the orbits and the dispersion around the same is not representative of the true errors for so few points. 
A\&A 511, A55 (2010)

Table 4. The different RV precision indicators on the RV std, TW Hya, and Gl 86.

\begin{tabular}{lcccc}
\hline \hline & External dispersion $[\mathrm{m} / \mathrm{s}]$ & Intra-Night Dispersion $[\mathrm{m} / \mathrm{s}]$ & Photon Noise $[\mathrm{m} / \mathrm{s}]$ & $(\mathrm{O}-\mathrm{C})[\mathrm{m} / \mathrm{s}]$ \\
\hline RV std & 5.77 & 7.03 & 6.48 & - \\
TW Hya & 54.57 & 12.12 & 12.10 & 7.93 \\
Gl 86 & 122.47 & 12.77 & 7.62 & 5.41 \\
\hline
\end{tabular}

Table 5. The probability of obtaining a dispersion of $5.77 \mathrm{~m} / \mathrm{s}$ on a set of 5 points characterized by dispersions in the range $5-10 \mathrm{~m} / \mathrm{s}$.

\begin{tabular}{cc}
\hline \hline$\sigma[\mathrm{m} / \mathrm{s}]$ & $P[\%]$ \\
\hline 5 & 84.1 \\
6 & 66.3 \\
7 & 49.9 \\
8 & 36.6 \\
9 & 26.6 \\
10 & 19.5 \\
\hline
\end{tabular}

A question that arises naturally is which factors limit the RV precision delivered by this method? This is difficult to evaluate mostly because the data set is small. However, on theoretical ground, some educated guesses can be made. The precision on each of the telluric lines central wavelength is not quantified on the HITRAN database from which the values were drawn. The lines are merely flagged as having a precision between 5 and $50 \mathrm{~m} / \mathrm{s}$. It is thus not surprising that a precision of $5 \mathrm{~m} / \mathrm{s}$ can be reached as a whole, but this might become the limiting factor if one pushes the $S / N$ to much higher values. The eventual existence of a systematic wavelength assignment error can also be a trivial source of error. On the other hand, atmospheric phenomena such as localized jet streams may imprint an $\mathrm{RV}$ on the reference lines themselves. However, its is hard to evaluate how strong these effects are when we integrate along the line of sight, up to the outer atmosphere. The conjugation of three factors point to $\mathrm{CO}_{2}$ features as some of the most stable in the atmospheric spectrum, though: 1) the abundance of $\mathrm{CO}_{2}, 2$ ) the constant volume mixing ratio of $\sim 300 \mathrm{ppmv}$ up to $80 \mathrm{~km}, 3$ ) the low absorption line intensities. When taken together, these properties reduce the impact of localized air mass movements on the RV. With the current set of data, we can only evaluate the short-term stability of the atmospheric lines. An extended data set with observations under very different atmospheric conditions is now under evaluation. The results will be the subject of a forthcoming paper (Figueira et al., in prep.).

Temperature and pressure changes are known to be one of the main causes of spectrograph's IP variations. It is important to note that CRIRES is cryogenic and thus stabilized in temperature. At the time of writing, the thermal stability of the most important components of the spectrograph is $\sim 0.05 \mathrm{~K}$ for the prism, $0.2 \mathrm{~K}$ for the grating, and $0.3 \mathrm{~K}$ for the Three-Mirror-Astigmat. As a consequence, the IP remains constant and its impact on RV measurements is reduced. Another possible line profile variation comes from atmospheric phenomena. The eventual variations are seen by both our reference and target spectra. The drawback is that this shows that typical bisector analysis cannot be used in a straightforward way in stellar spectra, because one must remove the effect of the variation of atmospheric lines' bisector beforehand. It is then not a surprise that the bisector shows no correlation with the RV. Still, the low number of points and the possibility of atmospheric profile variations makes this diagnosis very weak. The data do not provide for a finer analysis, which is not within the scope of this paper.
The contribution of photocenter errors was negligible in our error budget, but that might not be the case if the seeing becomes very low, as experienced by Bouchy et al. (2005). However, our situation is fundamentally different, because our reference lines will be affected by these photocenter movements in the same way as the object lines. A simple way to reduce this effect is to use a fiber entrance, providing for an homogeneous illumination over a wide range of seeing conditions.

That PHOENIX-derived masks lead to a lower precision than masks derived from the data themselves is insightful on its own. It states that the line's position precision as calculated by stateof-the art models is lower than the one that can be measured from high $S / N$ spectra. Some RV determination methods are more sensitive to this fact than others. In particular, those that obtain the RV by searching for a match between stellar models and observed spectra struggle against an additional and non-negligible source of noise.

While the application of gas cells on general-purpose slit spectrographs such as CRIRES is very tempting, their yielded precision still needs to be assessed. As already discussed by Mahadevan \& Ge (2009), using the gas cell as a simultaneous reference superimposed on the stellar spectrum is limited by the definition of the stellar continuum. In the case of M stars, one of the main drivers for RV in the IR, the continuum is shaped by a dense forest of unresolved spectral lines. These features are convolved with the atmospheric transmission, which is variable through time. These factors together make RV determinations much more demanding, time, and photon-consuming and, in many cases, degenerated. In our particular approach, such a poor continuum definition would introduce systematic errors into the absorption lines wavelength assignment, which would propagate to the final calculated RV.

Several infrared spectrographs dedicated to RV measurements are being designed, such as NAHUAL (Martín et al. 2005) and SPIROU ${ }^{4}$. These spectrographs aim at covering a wide spectral range simultaneously from around 1 to $2.2 \mu \mathrm{m}$. If a gas cell on the main optical path is chosen as the calibration procedure, the yielded precision will vary locally as a function of stellar continuum definition and atmospheric subtraction quality. On the other hand, using this gas cell on a twin optical path with an homogeneous continuum source or other methods, such as a laser coupled to a Fabry-Perot resonant cavity, allows a simple avoidance of these problems. However, as discussed before, this approach is only possible in a fiber-fed, stable spectrograph.

\section{Conclusions}

We proved that RV measurements with a precision of $5-10 \mathrm{~m} / \mathrm{s}$ are within reach of CRIRES, while using carefully selected atmospheric features as a wavelength reference. This precision was obtained on an RV standard, HD 108309, over a time span of roughly one week. TW Hya RV variation in the IR is not compatible with its optical counterpart, showing that a stellar spot is the

\footnotetext{
4 http://www.ast.obs-mip. fr/article.php3?id_ article $=637$
} 
best explanation for the observed RV variations. This confirms our previously published results (Huélamo et al. 2008) with better precision and consistency. We also showed that the RV variation of a star harboring a planet, Gl 86, is reproduced well by CRIRES data with a precision that is compatible with photonnoise estimations and at the same noise level.

Using the Earth's atmosphere as a free gas-cell still needs to be fully assessed, and the outcome of these studies will, in all probability, shape the design of future dedicated IR spectrographs. To do so, more lengthy data sets spanning a longer timespan are required. However, while this method seems promising and able to compete with regular gas-cells approaches, it is not yet been proved that higher precision is possible. Strong limitations might arise below $5 \mathrm{~m} / \mathrm{s}$ steaming from sources such as the knowledge of the precise wavelength of telluric lines or their stability on long time scales.

Acknowledgements. P.F. would like to thank every researcher who contributed to the enthusiastic, fast-paced, and extremely friendly environments of both the Observatoire de Genève and ESO Santiago. This work was shaped by the passionate discussions it triggered. Support from the Fundação para Ciência e a Tecnologia (Portugal) to P.F. in the form of a scholarship (reference SFRH/BD/21502/2005) is gratefully acknowledged. N.C.S. would like to acknowledge the support by the European Research Council/European Community under the FP7 through a Starting Grant, as well as from Fundação para a Ciência e a Tecnologia (FCT), Portugal, through program Ciência 2007, and in the form of grants reference PTDC/CTE-AST/098528/2008 and PTDC/CTEAST/098604/2008. We thank the ESO Director Office for granting DDT observations. We also thank the anonymous referee for his/her valuable suggestions on how to improve the manuscript.

\section{References}

Bailey, J., Simpson, A., \& Crisp, D. 2007, PASP, 119, 228

Balthasar, H., Thiele, U., \& Woehl, H. 1982, A\&A, 114, 357

Baranne, A., Queloz, D., Mayor, M., et al. 1996, A\&AS, 119, 373

Barman, T. S., Hauschildt, P. H., \& Allard, F. 2005, ApJ, 632, 1132

Blake, C. H., Charbonneau, D., White, R. J., Marley, M. S., \& Saumon, D. 2007, ApJ, 666, 1198

Bonfils, X., Delfosse, X., Udry, S., et al. 2004, in Spectroscopically and Spatially Resolving the Components of the Close Binary Stars, ed. R. W. Hilditch,

H. Hensberge, \& K. Pavlovski, ASP Conf. Ser., 318, 286

Bouchy, F., Pepe, F., \& Queloz, D. 2001, A\&A, 374, 733

Bouchy, F., Pont, F., Melo, C., et al. 2005, A\&A, 431, 1105
Bretagnon, P., \& Francou, G. 1988, A\&A, 202, 309

Butler, R. P., Marcy, G. W., Williams, E., et al. 1996, PASP, 108, 500

Caccin, B., Cavallini, F., Ceppatelli, G., Righini, A., \& Sambuco, A. M. 1985, A\&A, 149, 357

Desort, M., Lagrange, A.-M., Galland, F., Udry, S., \& Mayor, M. 2007, A\&A, 473, 983

Eggenberger, A., Udry, S., \& Mayor, M. 2003, in Scientific Frontiers in Research on Extrasolar Planets, ed. D. Deming, \& S. Seager, ASP Conf. Ser., 294, 43

Endl, M., Cochran, W. D., Wittenmyer, R. A., \& Boss, A. P. 2008, ApJ, 673, 1165

Galland, F., Lagrange, A.-M., Udry, S., et al. 2005, A\&A, 444, L21

Hatzes, A. P., Guenther, E. W., Endl, M., et al. 2005, A\&A, 437, 743

Horne, K. 1986, PASP, 98, 609

Howard, A. W., Johnson, J. A., Marcy, G. W., et al. 2009, ApJ, 696, 75

Huélamo, N., Figueira, P., Bonfils, X., et al. 2008, A\&A, 489, L9

Kaeufl, H.-U., Ballester, P., Biereichel, P., et al. 2004, in SPIE Conf. Ser. 5492, ed. A. F. M. Moorwood, \& M. Iye, 1218

Livingston, W., \& Wallace, L., 1991, An atlas of the solar spectrum in the infrared from 1850 to $9000 \mathrm{~cm}^{-1}$ (1.1 to 5.4 micrometer), ed. W. Livingston, \& L. Wallace

Lovis, C., \& Mayor, M. 2007, A\&A, 472, 657

Lovis, C., \& Pepe, F. 2007, A\&A, 468, 1115

Lovis, C., Mayor, M., Pepe, F., et al. 2006, Nature, 441, 305

Mahadevan, S., \& Ge, J. 2009, ApJ, 692, 1590

Martín, E. L., Guenther, E., Barrado y Navascués, D., et al. 2005, Astron. Nachr., 326, 1015

Martín, E. L., Guenther, E., Zapatero Osorio, M. R., Bouy, H., \& Wainscoat, R. 2006, ApJ, 644, L75

Mayor, M., Pepe, F., Queloz, D., et al. 2003, The Messenger, 114, 20

Mayor, M., \& Queloz, D. 1995, Nature, 378, 355

Mayor, M., Udry, S., Lovis, C., et al. 2009, A\&A, 493, 639

Pepe, F., Mayor, M., Galland, F., et al. 2002, A\&A, 388, 632

Prato, L., Huerta, M., Johns-Krull, C. M., et al. 2008, ApJ, 687, L103

Queloz, D., Henry, G. W., Sivan, J. P., et al. 2001, A\&A, 379, 279

Queloz, D., Mayor, M., Weber, L., et al. 2000, A\&A, 354, 99

Rothman, L. S., Jacquemart, D., Barbe, A., et al. 2005, J. Quant. Spectrosc. Radiat. Transf., 96, 139

Sato, B., Toyota, E., Omiya, M., et al. 2008, PASJ, 60, 1317

Seifahrt, A., \& Käufl, H. U. 2008, A\&A, 491, 929

Setiawan, J., Henning, T., Launhardt, R., et al. 2008, Nature, 451, 38

Setiawan, J., Weise, P., Henning, T., et al. 2007, ApJ, 660, L145

Smith, M. A. 1982, ApJ, 253, 727

Snellen, I. A. G. 2004, MNRAS, 353, L1

Sousa, S. G., Santos, N. C., Israelian, G., Mayor, M., \& Monteiro, M. J. P. F. G. 2007, A\&A, 469, 783

Sozzetti, A., Udry, S., Zucker, S., et al. 2006, A\&A, 449, 417

Tody, D. 1993, in Astronomical Data Analysis Software and Systems II, ed. R. J. Hanisch, R. J. V. Brissenden, \& J. Barnes, ASP Conf. Ser., 52, 173

Wallace, L., Hinkle, K., \& Livingston, W. C. 1993, An atlas of the photospheric spectrum from 8900 to $13600 \mathrm{~cm}^{-1}$ (7350 to 11230 [angstroms]), ed. L. Wallace, K. Hinkle, \& W. C. Livingston 